\title{
« La sportive française » entre Révolution nationale et commandement allemand
}

"The French sportswoman" between national Revolution and German military command

\section{Fatia Terfous}

\section{(2) OpenEdition}

\section{Journals}

Édition électronique

URL : http://journals.openedition.org/clio/10850

DOI : 10.4000/clio. 10850

ISSN : 1777-5299

Éditeur

Belin

Édition imprimée

Date de publication : 31 décembre 2012

Pagination : 209-234

ISSN : 1252-7017

Référence électronique

Fatia Terfous, « « La sportive française » entre Révolution nationale et commandement allemand », Clio. Femmes, Genre, Histoire [En ligne], 36 | 2012, mis en ligne le 31 décembre 2014, consulté le 19 avril 2019. URL : http://journals.openedition.org/clio/10850 ; DOI : 10.4000/clio.10850 


\section{Varia}

\section{« La sportive française " entre Révolution nationale et commandement allemand}

Fatia TERFOUS

Nombre d'ouvrages traitent de la période de Vichy. Ses aspects politiques, économiques, juridiques, sociaux et culturels sont désormais bien connus ${ }^{1}$. Le domaine sportif a été également largement investi par les historiens ${ }^{2}$. Les récentes études sur les pratiques physiques et sportives des femmes et des jeunes filles, quant à elles, s'attachent principalement à l'action de Marie-Thérèse Eyquem ${ }^{3}$. Première femme nommée directrice des sports féminins au sein du commissariat général à l'éducation générale et aux sports (CGEGS) en 1940, Marie-Thérèse Eyquem conçoit une sorte de doctrine féminine. Elle oriente sa politique autour de la formation des cadres, de la construction d'installations matérielles nouvelles, de la recherche d'une collaboration entre les différentes fédérations et de la propagande sportive. Elle milite avec ardeur en faveur du sport féminin et obtient une certaine écoute de Jean Borotra (premier commissaire au CGEGS). À partir de 1942, elle exerce sa politique dans l'indifférence totale des pouvoirs publics alors

1 Azéma 1979; Faure 1989; Azéma \& Bédarida 1992, 1993 ; Laborie 1990 ; Paxton 1973 ; Peschanski 1997.

2 Arnaud \& Riodan 1998 ; Arnaud et al. 2002 ; Gay Lescot 1991; Rauch 1988 ; Delaplace, Treutlein \& Spitzer 1994.

3 Arnaud \& Terret 1996; Munoz 2002 ; Liotard \& Terret 2005; Saint-Martin \& Terret 2005 ; Terret 2005 ; Castan Vicente 2009. 
représentés par le Colonel Pascot (second commissaire au CGEGS)4. Si les études sur la politique de Marie-Thérèse Eyquem sont essentielles, les obstacles qui ont empêché sa mise en œuvre sont loin d'être négligeables ${ }^{5}$. Deux indicateurs, équipement sportif et formation des cadres, permettent de mesurer finement les écarts entre les intentions politiques et leurs réalisations. Par ailleurs, le morcellement du pays a des conséquences politiques essentielles puisqu'il amène l'installation de systèmes d'occupation différents sur le territoire: deux administrations militaires allemandes (en zone Nord avec le Commandement de Paris, et dans la zone rattachée avec celui de Bruxelles), un Commandant allemand des forces armées en zone Sud à partir de novembre 1942 (et un autre italien pour l'autre zone d'occupation), deux administrations civiles du Reich pour l'Alsace et la Moselle. Dans la Zone Interdite où les départements du Nord et du Pas-de-Calais sont rattachés au Commandement militaire allemand de Bruxelles $^{6}$, la gestion de l'Occupation ne fut pas identique par rapport au reste du territoire, ni $a$ fortiori la politique de persécution ou la manière de mener la répression?

\section{L'ambition de Vichy en matière sportive et féminine : doctrine de la Révolution nationale}

C’est parce que la défaite de 1940 est synonyme de défaite physique que le maréchal Pétain intègre dans la Révolution nationale une

$4 \quad$ Eyquem 1967.

5 Le corpus est construit à partir des archives du Centre d'accueil et de recherche des archives nationales (séries F44, F17 et 69 AJ), de l'Institut national du Sport et de l'Éducation physique (INSEP), de la Fédération sportive et culturelle de France (FSCF), des archives départementales du Nord et du Pas-de-Calais. Les sources écrites sont complétées par des sources orales, des entretiens semidirectifs réalisés auprès d'une monitrice d'éducation physique et sportive et d'une institutrice du Pas-de-Calais ont été aussi réalisés.

6 Les départements du Nord et du Pas-de-Calais, rattachés au commandement militaire allemand de Belgique et du nord de la France, sont classés Zone Interdite en novembre 1940. La mise en vigueur de la législation française dans cette zone est soumise avant toute exécution à l'administration du général Niehoff et plus généralement à l'OFK 670 de Lille. Dejonghe \& Le Maner 1998 ; Fontaine 2009.

7 Thiery 2007. 
rénovation physique et morale. Sous Vichy, le CGEGS a toute compétence pour organiser et gérer le sport et l'éducation physique. L'idée maitresse qui a présidé à l'élaboration de la loi du 20 décembre 1940 relative à l'organisation sportive, publiée au Journal Officiel le 8 avril 1941, est de concilier le principe d'autorité avec le maintien d'une large initiative privée. Avec la nouvelle loi, cette initiative privée se manifeste à la base, c'est-à-dire au sein de l'association sportive. À cet échelon, l'autorité s'exerce uniquement sous forme d'un contrôle. L'initiative privée subsiste également aux échelons supérieurs (comités régionaux, fédérations) mais à ces stades, qui concernent l'orientation sportive générale du pays, l'autorité intervient progressivement. Il faut tout de même attendre le 19 novembre 1941 pour la publication du décret d'application de cette loi. L'ensemble des textes porte l'appellation de Charte des Sports mais la loi du 20 décembre 1940 est déjà connue sous la même désignation ${ }^{8}$. La Charte des Sports, qui inaugure un régime de liberté contrôlée, fixe des responsabilités. Elle prévoit des sanctions. Elle assure, au sein des associations sportives, "l'autorité, l'ordre et le travail »?. Elle doit permettre aux organismes sportifs, et en particulier aux associations, d'accomplir la mission qui leur est désormais confiée dans le pays. Les associations, en plus d'être des centres d'acquisition de technique sportive, doivent être désormais pour les jeunes «des foyers d'éducation, où ils développeront leurs qualités morales : courage, ténacité, loyauté, maîtrise de soi, esprit de sacrifice». La charte comprend une doctrine nationale dans laquelle l'acquisition de la santé, la formation du caractère et de l'énergie morale et physique, le goût de l'action et le sens de l'altruisme «sont prônés avant toute chose ». La doctrine nationale présente les activités préconisées et leur application mais aussi la formation et les missions des cadres ${ }^{10}$.

\footnotetext{
Callède $1998: 69$.

9 Journal Officiel, 20 décembre 1940. Loi relative à l'organisation sportive. Les citations de la Charte proviennent de ce JO.

10 Callède 1998 : 68.
} 
La direction du sport féminin est confiée à Marie-Thérèse Eyquem $^{11}$. Cette dernière œuvre en direction du redressement national bien connu et organise le sport féminin autour de valeurs propres, validées par des considérations médicales, sociales et morales, devant se garder de tout risque de masculinisation, d'excès, de compétition et d'exhibition spectaculaire. Elle envisage de corriger les erreurs commises par le passé et plus particulièrement l'orientation politique du sous-secrétariat d'État à l'organisation des sports et des loisirs, confié à Léo Lagrange en 1936. L'absence de contrôle public sur le sport féminin, les "excès» de certaines sportives comme Violette Morris ${ }^{12}$, l'image de la sportive d'avant-guerre qui pratique le football et la barrette, qui sont à la base des nombreuses plaintes « des dirigeants, monitrices-moniteurs et des parents $»^{13}$, justifient pour Marie-Thérèse Eyquem une politique nouvelle. Elle ambitionne de jeter les bases d'une organisation tendant à réduire au maximum les principaux dangers résultant de l'activité physique mal dirigée (mauvais dosage, surentraînement, masculinisation, surmenage, troubles articulaires, cabotinage) et à éviter toute forme d'excès et de brutalité de façon à réaliser « un sport équilibré, mesuré, simple, en un mot véritablement féminin $»^{14}$. Marie-Thérèse Eyquem établit une sorte de doctrine sportive dans laquelle l'éternel féminin, largement décrit par Muel-Dreyfus ${ }^{15}$, permet de définir les espaces et les pratiques qui conviennent aux femmes.

11 L'analyse de « la sportive française » selon le CGEGS a été construite à partir des sources officielles: Tous les sports, organe de presse officiel du CGEGS (19401944), FSCF ; publications, revues officielles du CGEGS, courriers, plans, circulaires, rapports des archives nationales du CARAN (séries F44, F17 et 69 AJ) ; journaux officiels consultés aux archives départementales du Nord.

12 Ruffin 2004. Violette Morris représenta la France dans les compétitions sportives entre les années 1912 et 1935. Championne de France et du monde des lancers, footballeuse internationale, également brillante en cyclisme, boxe et automobile, elle devient une figure héroïque controversée en raison, notamment, de sa bisexualité.

13 Tous les sports, 2 août 1941 (FSCF).

14 Tous les sports, 12 juillet 1941.

15 Muel-Dreyfus 1996. 
Elle place les fédérations féminines sous le giron des fédérations masculines en raison "d'une plus grande fermeté des hommes $»^{16}$. Elle souhaite que les femmes soient représentées au sein des fédérations sportives ainsi que dans les comités régionaux et les commissions techniques. Cependant, elle précise que le travail commun réalisé entre les hommes et les femmes ne doit pas faire disparaitre les différences existantes ni nuire à la santé et à l'esthétique des femmes ${ }^{17}$. Ainsi, les jeunes filles peuvent pratiquer les sports ou l'éducation physique au sein des 13 fédérations ayant des sections féminines, mais aussi auprès de l'Union des sociétés de gymnastique de France, de la Fédération gymnique et sportive des patronages de France et de la Fédération sportive et gymnique du travail. Les unions pratiquent la doctrine nationale dans son intégralité (éducation physique, initiation sportive, éducation rythmique, sports) et se chargent de former leurs cadres. Les fédérations de football, de barrette, de cyclisme et des sports de combats ne possèdent pas de sections féminines. Marie-Thérèse Eyquem voit dans ces pratiques un obstacle au développement du sport féminin raisonné, sain et gracieux. À l'inverse, elle promeut largement la rythmique pour les femmes et les jeunes filles car «leur attitude sans noblesse n'imposerait pas aux hommes le respect qui fait naître l'admiration, ses mouvements étriquées ou maladroits la rendraient ridicule $»^{18}$. Comme la décence des sportives évoluant sur le stade et les plateaux d'éducation physique est une de ses préoccupations majeures, elle impose une tenue vestimentaire: culottes et sandales sont désormais obligatoires. Par ailleurs, elle conseille aux femmes d'aborder les compétitions uniquement si leur organisme le permet (organisme particulièrement solide, équilibré, doué) et si elles ne nuisent ni à leur santé, ni à leur vie familiale et professionnelle. Enfin, elle exige un entraînement non mixte pour empêcher l'immoralité et

16 Tous les sports, 12 juillet 1941, «Marie-Thérèse Eyquem nous parle du sport féminin » (FSCF).

17 Ibid.

18 Tous les sports, 26 juillet 1941, «Le sport et la femme: le rythme, une école de grâce » (FSCF). 
éviter le surentraînement. Pour les mêmes raisons, elle envisage de limiter les manifestions sportives ${ }^{19}$.

Finalement, Marie-Thérèse Eyquem établit une doctrine sportive propre aux femmes et aux jeunes filles :

Robustes, énergétiques, mais demeurées essentiellement femmes, elles seront des épouses et des mères dignes de former les générations nouvelles, notre seul salut. Tâche sublime, la plus belle. Elles serviront leur pays en s'aimant les unes les autres malgré les différences de conviction, de milieu social, d'instruction; en donnant l'exemple de l'union et de la bonté ; en marquant leur attachement indéfectible à la patrie ${ }^{20}$.

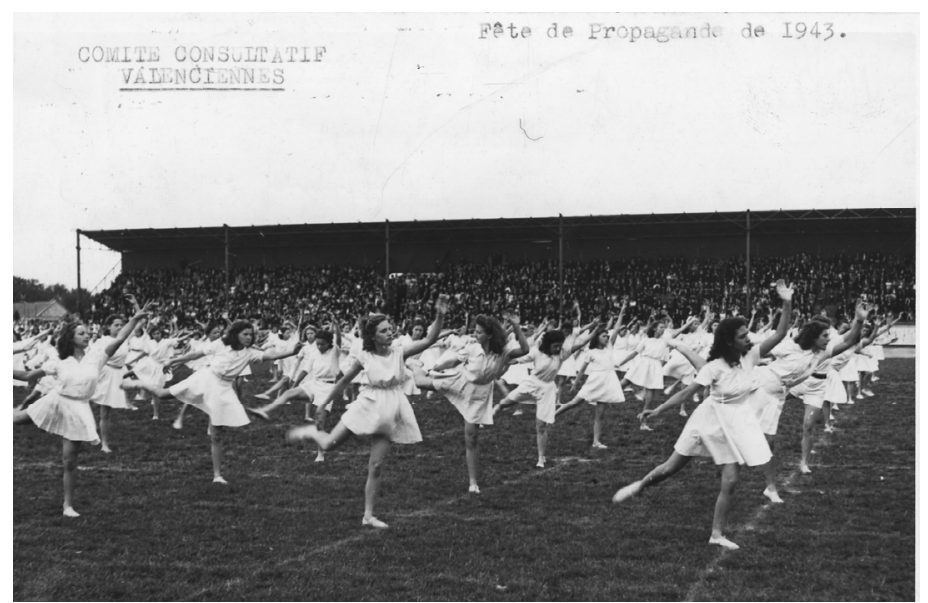

A.M.V. Fête de propagande 1943 (1 W 1004).

(C) Archives municipales de Valenciennes - Cliché C. Gérard

Les discours de Marie-Thérèse Eyquem, en avançant des considérations physiologiques, morales et sociales, affirment avant tout les choix politiques de la Révolution nationale. Ils montrent un resserrement et une uniformisation des pratiques sportives. La culture physique sous ces formes appropriées, souvent gymniques, est largement valorisée pour ses finalités esthétiques (féminité, grâce,

19 Ibid.

20 Tous les sports, 17 janvier 1942, «Marie-Thérèse Eyquem : comment est organisé le sport féminin » (FSCF). 
souplesse) et morales. La saine préparation des jeunes filles et des femmes à la maternité est également récurrente dans ses écrits. Cependant la conception utilitaire du «sport» pour favoriser la maternité et l'exigence de grâce et de beauté n'est pas nouvelle. Elle forme un consensus social et politique durant toute la première moitié du $\mathrm{XX}^{\mathrm{e}}$ siècle ${ }^{21}$. La doctrine sportive féminine de Marie-Thérèse Eyquem renforce les stéréotypes de genre et s'inscrit dans un processus déjà antérieur à la politique de Vichy: la chute de la natalité, le développement de l'hygiénisme, le spectre de la dégénérescence, l'empreinte du catholicisme, l'évolution lente des mentalités. C'est ce qu'elle constatait, non sans appréhension :

Il est de mon devoir d'attirer l'attention des sportives sur le fait que le mouvement sportif féminin qui, après la guerre de 1914 prenait de si large proportions, a été freiné. La même menace pèse sur notre mouvement actuel si nos sportives ne restent pas mesurées et unies. Les unissent et les uniront plus que jamais le même amour d'une jeunesse saine et forte, le désir d'une formation physique, qui se manifeste extérieurement par une belle tenue, une formation morale dont la bonne éducation est un des signes les plus souhaitables. Les unit surtout la communauté de race et d'histoire qui crée l'irremplaçable communion ${ }^{22}$.

Pour Laurence Munoz ${ }^{23}$, l'action de Marie-Thérèse Eyquem trouve son point d'appui dans son parcours associatif. Ses fonctions de secrétaire nationale du Rayon Sportif Féminin, qui regroupe les patronages des sœurs de Saint-Vincent-de-Paul, auraient pesé dans son embauche au sein du commissariat. La mère de Marie-Thérèse Eyquem découvre une offre d'emploi de rédactrice émanant du CGEGS. Jugée sur dossier, elle est embauchée le 17 août 1940. Considérée comme en phase avec le comportement d'une frange de catholiques largement acquis aux idées de la Révolution nationale, son embauche au CGEGS ne semble pas, pour Laurence Munoz, revêtir un caractère politique. Selon l'auteure, il s'agit moins d'un comportement requis que d'une opportunité de carrière pour cette

21 Louveau $2006: 133$.

22 Tous les sports, 15 novembre 1941, «Marie-Thérèse Eyquem : Sportives restez toujours unies » (FSCF).

23 Munoz $2002: 66-72$. 
jeune femme de 27 ans. Florys Castan Vicente ${ }^{24}$, qui retrace la trajectoire de la militante pour le sport féminin, montre également comment son éducation catholique et son expérience au sein du Rayon Sportif Féminin ont contribué à définir ses conceptions du sport et de l'éducation physique. Selon l'auteure, la définition du sport féminin comme une activité physique devant permettre aux femmes de développer la grâce et la féminité dans un cadre de pratique «moralisé », que semble partagée Marie-Thérèse Eyquem, est souvent liée à la menace que fait peser le sport sur le statut de mère et d'épouse des femmes.

Au service du sport féminin mais usant des stéréotypes les plus répandus, l'action de Marie-Thérèse Eyquem sous Vichy est différemment interprétée. Pour Florys Castan Vicente, elle est comprise à la fois comme une allégeance certaine aux thèses du moment et comme novatrice et émancipatrice :

La théorie d'une action novatrice, permettant aux femmes de pratiquer n'importe quel sport contredit les sources. En revanche, l'idée que Vichy ait favorisé une émancipation féminine sans le vouloir, par l'intermédiaire d'une image positive de la «sportive-femme» parait crédible, bien que les archives manquent ${ }^{25}$.

En 1944, Marie-Thérèse Eyquem indiquait pourtant :

Première femme chargée des sports féminins par Jean Borotra au CGEGS, j'organisais en 1943, une "Journée Nationale de la Sportive » qui groupait 50000 sportives le même jour sur une trentaine de stades, dans une indifférence totale des Pouvoirs Publics représentés alors par le Colonel Pascot ${ }^{26}$.

On mesure ici toute la fragilité de la doctrine sportive féminine. Les installations matérielles et la formation de cadres sont deux indicateurs pertinents pour révéler la difficile mise en œuvre de la politique sportive élaborée par Marie-Thérèse Eyquem.

24 Castan Vicente 2009.

25 Castan Vicente 2009 : 54.

26 Caillois $1967: 1826-1838$. 
Les moyens d'une politique :

l'équipement sportif et les cadres de Vichy

Annoncé comme une priorité du CGEGS et conditionnant la réalisation de la doctrine sportive féminine, l'équipement sportif est le premier indicateur retenu pour mesurer le degré d'application de la politique sportive de Vichy ${ }^{27}$. Le 13 novembre 1940, le CGEGS bénéficie d'un crédit de deux milliards de francs pour le développement de l'équipement sportif français ${ }^{28}$. À ce titre, les slogans et les affiches abondent pour rappeler la nécessité de doter la France d'un équipement sportif suffisant ${ }^{29}$. Le commissariat propose des subventions aux collectivités soucieuses du développement physique de leurs administrés en particulier pour l'achat de terrains. Celles-ci sont comprises entre $40 \%$ et $60 \%$ de la valeur vénale du lieu à acquérir. En revanche, dans le cas d'aménagements de terrains loués, la subvention ne peut dépasser 100000 francs. Pendant un délai de cinq ans, à compter de la publication de la loi du 18 juillet 1941 tendant à faciliter l'exécution d'urgence des travaux d'équipement sportif, les départements et l'État peuvent poursuivre l'expropriation pour cause d'utilité publique, des propriétés nécessaires à l'établissement de toutes installations d'éducation générale ou sportive ${ }^{30}$. Les taux de subvention les plus élevés sont destinés aux réalisations promettant le développement de l'éducation physique et des sports de base (athlétisme et natation). Les associations sportives subventionnées prennent l'engagement d'entretenir les installations et de les mettre à la disposition des enfants des écoles et autres associations sportives. En janvier 1942, dans chaque commune sont créés des comités consultatifs locaux, établissant les conditions d'utilisation des installations sportives

27 L'analyse de la politique en faveur de l'équipement sportif a été construite à partir des archives de l'INSEP (brochures du CGEGS, correspondances, circulaires, acquisitions et réquisitions des terrains de sport par les troupes d'occupation), des archives nationales du CARAN (séries F44), des archives départementales du Nord (séries 42W), des archives municipales d'Arras (série 3R) et de Douai (série 3D).

28 Journal Officiel, 6 août et 4 septembre 1941.

29 Brochure du CGEGS et Journal Officiel du 6 août et du 4 septembre 1941.

30 Journal Officiel, 4 septembre 1941. 
communales et privées ${ }^{31}$. Cependant, en raison du manque de main d'œuvre et de matériaux de construction, les autorités allemandes prévoient en octobre 1942 qu'à l'avenir seules les constructions particulièrement nécessaires et vitales seront entreprises ${ }^{32}$. Dès mars 1942, Jean Borotra écrit :

les installations complètes sont infiniment difficiles à réaliser, par suite du manque de matières premières. Nous n'avons pu en l'espace de 15 mois, subventionner que 500 projets d'installations sportives définitives, ce qui n'est rien en regard des 36000 communes de France ${ }^{33}$.

Face aux insuffisantes réalisations de son prédécesseur, le colonel Pascot prend de nouvelles mesures censées tenir compte de la pénurie de matériaux, de main-d'œuvre et de la limitation des grands travaux autorisés. Des subventions de $40 \%$ à $60 \%$ du prix sont données aux collectivités pour l'acquisition de terrains. Un ordre d'urgence dans l'exécution des travaux et une décentralisation des études et des crédits sont même réalisés. À la fin de l'année 1943, le bilan des activités du Commissariat général pour l'équipement sportif montre que 2000 projets ont été déposés et étudiés. Ils portent sur 4 milliards 700 millions de francs de travaux. Sur ce nombre, 1350 projets définitifs (pour 925 millions) et 10000 aménagements sommaires (pour 600 millions) ont été crédités. En outre, 450 projets de baignades aménagées ont reçu 13 millions à titre de subvention. Enfin, il est prévu l'équipement de 55 piscines, bassins et centres du Commissariat général. Pour ces derniers une dépense de 265 millions est envisagée. Néanmoins, au regard des efforts déployés par l'Allemagne et l'Angleterre, le colonel Pascot n'est toujours pas satisfait des résultats obtenus en matière d'équipement sportif ${ }^{34}$.

31 Comité consultatif local de l'EGS, AM d'Arras, 3R4. Seules 13 chefs d'établissements de sexe féminin et une présidente d'association sportive font partie du Comité consultatif local d'EGS d'Arras.

32 Lettre de l'OFK 670 de Lille au préfet régional du Nord, 14 octobre 1942, ADN, 1W247.

33 Jean Borotra, Conférence d'information VII, l'Éducation Générale et les Sports, INSEP, p. 29

34 Tous les sports, 25 décembre 1943 (FSCF). 
La formation des cadres démontre également le décalage entre les intentions politiques et la réalité des mesures prises ${ }^{35}$. Dans la doctrine nationale un encadré à l'attention des éducateurs précise l'adaptation de la doctrine à l'éducation générale féminine :

La jeune fille a besoin d'un entraînement méthodique qui assurera son développement physique et celui des qualités de caractère, en s'adaptant à la puberté, en la préparant pour l'avenir à la maternité : mission spéciale de la femme 36

D'ailleurs Marie-Thérèse Eyquem souhaite que les monitrices se substituent graduellement aux moniteurs. Pour elle, il est préférable que les jeunes filles et les femmes aient un personnel féminin d'une moralité irréprochable, informé des particularités de l'organisme féminin dont il aura la charge. Elle souligne qu'à l'école, les jeunes filles doivent bénéficier de l'action éducatrice exercée par les institutrices ou les maitresses d'éducation générale. La monitrice d'association doit, selon elle, poursuivre l'action commencée par le professeur de l'établissement scolaire, adaptant simplement la méthode nationale à l'âge, au temps disponible, au lieu, à la mentalité ambiante et au milieu. On voit ici comment les cadres de Vichy doivent assurer un continuum entre les pratiques scolaires et extrascolaires. Sous Vichy, les cadres peuvent être formés au sein de trois structures ${ }^{37}$ : les centres régionaux d'éducation générale et sportive (au nombre de quinze avec subdivisions masculines et féminines), le centre national d'éducation générale et sportive ${ }^{38}$ et le

35 L'analyse de la formation des cadres sous Vichy a été construite à partir des archives de l'INSEP et des archives nationales au CARAN (série F44): répertoires des textes réglementaires à l'usage des maitres, résumés des conférences faites à l'occasion des stages d'éducation générale, guides des maitres d'Éducation générale et sportive (EGS) et comptes rendus des stages organisés par l'Institut national d'éducation générale et les centres régionaux d'EGS.

36 Journal Officiel, 20 décembre 1940. Loi relative à l'organisation sportive. La doctrine nationale d'éducation générale et sportive.

37 Journal Officiel, 26 mars 1941. Loi du 21 mars 1941.

38 Le centre national d'éducation générale et sportive comprend l'Institut national d'éducation générale et sportive (chargé de la formation des inspecteurs de l'éducation générale et des sports et des maîtres d'éducation générale) et l'école nationale d'éducation physique et sportive (chargée de la formation des professeurs d'éducation physique et sportive). 
collège national des moniteurs et athlètes d'Antibes (chargé de la formation des moniteurs d'éducation physique et sportive).

Dans les classes élémentaires et établissements secondaires, les professeurs d'éducation physique et sportive et moniteurs assurent l'éducation physique. Les instituteurs et les institutrices, chargés des classes élémentaires, donnent eux-mêmes cet enseignement à leurs élèves. Si c'est au chef d'établissement que revient le choix du maitre d'éducation générale parmi les professeurs des disciplines intellectuelles, l'Institut national d'éducation générale et des sports, dont le programme débute le 20 novembre 1942, assure leur formation. En effet, l'Institut propose de présenter aux maitres d'éducation générale des données théoriques sur l'éducation physique, la physiologie de l'enfant, de l'adolescent, du jeune homme (puberté, nutrition, sexualité) et des méthodes de pédagogie moderne (méthodes françaises et étrangères, histoire des sports). Le programme vise également à donner un complément d'information sur la pédagogie de l'éducation physique et des sports ainsi que sur les techniques sportives (séances de démonstration dans les établissements et sur les stades). Son intention, au cours des premières semaines d'activité,

n'est pas de fixer en une méthode rigide, ignorante de la réalité permanente et des difficultés momentanées mais de mettre en commun la compétence, l'expérience et la bonne volonté de tous, pour réaliser un fructueux travail d'équipe ${ }^{39}$.

Chaque stage organisé à l'Institut national d'éducation générale confère aux maittres et aux maîtresses des pratiques spécifiques. En dehors du programme commun, l'accent est mis pour les maitresses sur la gymnastique harmonique et rythmique (conférences et démonstrations d'Irène Popard) et sur l'adaptation nécessaire des leçons d'initiation sportive (pour les filles : courses de vitesse, lancer, volley-ball) ${ }^{40}$. Ainsi, à partir de 1942, l'Institut propose aux maittres d'EGS des stages de formation dans lesquels seules les maitresses

39 Feuille d'information. 3 décembre 1943, Revue Éducation Générale et Sports. AN, CARAN, F44/42.

40 Programme du stage des maitres d'EGS du 30 août au 11 septembre 1943, AN, CARAN, F44/41. 
assistent aux conférences et démonstrations de gymnastique rythmique et harmonique d'Irène Popard ${ }^{41}$.

Les centres régionaux d'éducation générale et sportive sont destinés à former les instituteurs-institutrices et les moniteursmonitrices qui ont la charge de l'éducation sportive des élèves du primaire. L'arrêté du 16 août 1941 fixe à un mois la durée des stages d'éducation générale et sportive. Le CGEGS désigne, quant à lui, le contenu de formation et les centres qui reçoivent les stagiaires. Les candidats dont l'activité et les connaissances sont jugées satisfaisantes et qui obtiennent la moyenne au cours des épreuves reçoivent le diplôme élémentaire ${ }^{42}$. Le 16 mars 1942, une nouvelle certification parait dans le Journal Officiel de l'État français. En plus d'obtenir la moyenne sur l'ensemble des notes, le travail, la conduite à l'institut et le stage pédagogique sont évalués ${ }^{43}$. Le contenu de formation des instituteurs, institutrices, moniteurs et monitrices est identique. Il comprend les activités d'éducation générale indiquées par le CGEGS.

La formation des professeurs d'éducation physique est assurée au sein de l'école nationale d'éducation physique et sportive. De nouvelles instructions pour le concours de recrutement des professeurs sont entérinées en 1942. Elles s'inspirent des programmes d'activités d'éducation générale du CGEGS. Les candidats doivent désormais commenter en détail les instructions du 1er juin 1941, le Guide abrégé du moniteur et de la monitrice de Georges Hébert mais aussi adopter obligatoirement la méthode naturelle pour les exercices de pédagogie pratique. Les programmes limitatifs relatifs aux épreuves écrites des matières scientifiques (anatomie, physiologie, hygiène) sont seuls

41 Dans le programme du stage des maitres d'EGS du 30 août au 11 septembre 1943, les leçons d'initiation sportive sont dites "adaptées aux caractéristiques des filles » et comprennent des courses de vitesse, lancer et volley-ball, AN, CARAN, F44/41.

42 Journal Officiel, 3 septembre 1941. Au cours de leur formation, les élèves reçoivent des notions élémentaires de physiologie et d'hygiène sportive. Ils pratiquent la gymnastique corrective, les travaux manuels et agricoles, le chant choral, les activités de plein air et de la vie des camps, les jeux, le secourisme et l'éducation physique et sportive selon les principes de la méthode naturelle.

43 Journal Officiel, 19 mars 1942. 
conservés. Le concours d'admission ${ }^{44}$ et l'admission à l'année préparatoire $^{45}$ comprennent des épreuves spécifiques pour les candidates. Par décret du 31 décembre 1943, le certificat d'aptitude à l'éducation physique est remplacé par le certificat d'aptitude à l'enseignement de l'éducation physique et sportive. Le certificat est délivré aux candidates qui satisfont aux épreuves de l'examen préliminaire (épreuves éliminatoires et épreuves non cotées de natation et d'athlétisme) et de l'examen final (épreuves écrites, orales et pratiques). Pour être reçue, la candidate doit obtenir la moyenne des points sur l'ensemble des épreuves, soit un total minimum de 400 points sans note éliminatoire ${ }^{46}$. L'existence d'un enseignement spécifique et différent entre les élèves-professeurs masculins et féminins sous le régime de Vichy ne doit pas surprendre. Pour l'examen préliminaire les épreuves pratiques sont les mêmes pour les candidats et les candidates (assouplissements, course de vitesse, course de demi-fond, saut, lancer, grimper, natation et une huitième épreuve au choix), même si dans les épreuves athlétiques les distances des courses et les poids des engins pour les lancers sont moindres pour les femmes. Enfin, contrairement aux candidats, les candidates ne peuvent pas, parmi les activités proposées pour la huitième épreuve, choisir l'haltérophilie et certains agrès (cheval d'arçon). Dans l'épreuve pédagogique de l'examen final, le rugby, le football et les sports de combat sont exclus du programme pédagogique des candidates et sont remplacés par la gymnastique harmonique et rythmique. Les choix éducatifs faits par le gouvernement de Vichy vont dans le sens d'une séparation, via les pratiques, des rôles sociaux en fonction des sexes. En conséquence, la direction de l'école

44 Tous les sports, 31 janvier 1942, «Le nouveau programme du professorat» (FSCF). Les candidates subissent des épreuves de deuxième catégorie (gymnastique rythmique, danses folkloriques), une épreuve de pédagogie pratique (chant, travaux manuels, rythmique), une interrogation sur un exercice ou sur l'observation d'un élève en action, des épreuves de travail collectif (exercice gymnique d'ensemble, exercices rythmiques et danses).

45 Les épreuves libres pour les candidates sont: saut de cheval, équilibre, jonglage, disque, escrime, danse rythmique et folklorique, natation.

46 Ministre de l'Éducation nationale. CGEGS (1944). Professorat d'EPS, textes officiels, programme. INSEP. 
normale d'éducation physique encourage et favorise une différenciation de la formation des professeurs d'éducation physique ${ }^{47}$.

Le collège national des moniteurs et athlètes d'Antibes (CNMAA) se charge «exceptionnellement» de la formation des moniteurs et monitrices avec deux autres centres d'information. Le premier situé à Paris forme, du 12 janvier au 8 février 1941, 150 moniteurs sélectionnés. Le second, en Zone Occupée, doit à partir de mars 1941, former un nombre non déterminé de moniteurs ${ }^{48}$. Pour le concours d'admission au CNMAA, les candidates effectuent des épreuves spéciales et font l'objet d'un classement particulier. La première série d'épreuves comprend des courses, des lancers, un grimper à la corde et une épreuve de natation. La deuxième série est un examen de culture générale. La troisième série, de classement, comprend des épreuves écrites, physiques et orales ${ }^{49}$. Au CNMAA de ChâtenayMalabry, la section féminine est préparée à tous les exercices de la méthode nationale et initiée aux sports. Les femmes et les jeunes filles de France doivent bénéficier du «précieux enseignement des vertus physiques et morales reçues par les monitrices elles-mêmes lors de leur formation ${ }^{50}$. En conséquence, «la pratique des exercices physiques n'exclut pas la grâce, l'élégance et la souplesse $»^{51}$. D'ailleurs, le programme du stage de formation normale de la section féminine, qui a lieu du 12 au 17 juin 1944, inclut la gymnastique harmonique en plus des travaux dirigés, des études, de la biologie, de la pédagogie pratique corrective, de l'éducation physique, des sports, des agrès, du plein air, de l'initiation sportive et de la natation ${ }^{52}$. Si le 22 mars 1941, le nombre des emplois de moniteurs chefs est fixé à 200 et à 1000 pour les professeurs d'EPS ${ }^{53}$, le CNMAA aurait formé de janvier à août 1941,

47 Lebrun \& Léziart 2005.

48 Grand Écho du Nord, 20 novembre 1940.

49 Journal Officiel, 4 juillet 1942. Décret n¹9368 du 1er juillet 1942.

50 Boulat P. non daté. La section féminine du CNMA. Revue Stade. ADN, $1 \mathrm{~W} 4483 / 10$.

51 Ibid.

52 Centre national d'éducation générale, CNMA, programme du stage de formation normale de la section féminine du 12 au 17 juin 1944. AN, CARAN, F 44/41.

53 Journal Officiel, 23 mars 1941. Loi n¹ 294. 
150 moniteurs chefs, 180 moniteurs et 320 moniteurs débutants. La même année, "voici à peu près comment se chiffre le résultat de l'effort sportif féminin: 6000 monitrices d'éducation physique et sportive nouvellement formées $\gg{ }^{54}$.

Même s'ils ne sont pas une création du CGEGS, les instituts régionaux d'éducation physique, créés dans les universités sous la direction des facultés ou des écoles de médecine, doivent également participer à la formation des cadres de Vichy. Ils sont à la fois des centres de spécialisation pour les étudiants en médecine, des centres de préparation pour les candidats au certificat d'aptitude à l'enseignement de la gymnastique, des centres d'étude appliquée à la recherche des meilleures méthodes pédagogiques et à leur adaptation aux différents milieux et professions. L'analyse des rapports d'activité des instituts régionaux de Rennes, Poitiers, Caen, Bordeaux, Besançon, Dijon et Lille adressés au CGEGS pour l'année 1940-1941 et 1941-1942 montre que huit professeures d'EPS sont formées (respectivement une à Rennes, Poitiers, Caen, Nancy, Dijon et trois à Bordeaux) contre dix neuf professeurs ${ }^{55}$.

D'après le CGEGS, entre 1941 et 1943, le personnel enseignant serait passé de 608 à 1059 professeurs d'EPS et de 466 à 935 moniteurs d'éducation physique. En 1943, dans les neuf centres académiques, les stages d'éducation générale et sportive auraient été suivis volontairement par 4000 instituteurs et institutrices, 3500 élèves-maitres et maitresses, 350 maîtres et maîtresses, 78 élèves des écoles normales supérieures et 1200 professeurs et moniteurs délégués ${ }^{56}$. Si ambitieuse soit-elle, la politique sportive de Vichy fut contrariée. Les moyens de cette politique furent rapidement rattrapés par les contradictions internes, les réalités de guerre, les occupants, les populations locales et certains événements. Les problèmes de ravitaillement, le pillage des matériaux de construction, les dégradations des installations traduisent sinon une résistance, du moins une indifférence des populations sinistrées. Si participation à la

\footnotetext{
54 Tous les sports, 15 novembre 1941, « Marie-Thérèse Eyquem : Sportives françaises restez toujours unies » (FSCF).

55 AN, CARAN, F44/40.

56 Tous les sports, 25 décembre 1943, «Le bilan général de 1943 » (FSCF).
} 
politique sportive il y a, les restrictions, la pénurie, la sousalimentation, les bombardements, le manque de matériel, la réduction des moyens de communication et les autorités allemandes contrarient les volontés des autorités françaises. Pour le mouvement sportif féminin, les méthodes, les modes de recrutement, l'attitude des cadres, l'accès limité des femmes et des jeunes filles aux installations, les inquiétudes des autorités catholiques locales soucieuses de la moralité des pratiquantes, les réticences des éducatrices et des parents réduisent encore de manière significative la fabrication de la « sportive française » telle que Marie-Thérèse Eyquem l'entendait.

Les obstacles de la politique sportive : contradictions internes, réalités de la guerre, occupants, populations, événements

L'occupation partielle du territoire et son découpage en zones relevant d'autorités politiques et administratives différentes retardent la mise en application des directives relatives à l'équipement sportif. En 1941, le Nord Pas-de-Calais est classé premier département de la Zone Occupée pour l'aménagement de terrains, mais le préfet du Nord note que 121 communes de son département ont présenté des projets qui sont restés lettre morte ${ }^{57}$. La loi d'expropriation des terrains pour cause d'utilité publique du 18 juillet 1941, qui vise à faciliter l'exécution des travaux d'équipements sportifs, n'atténue pas les difficultés. Les maires approuvent le maintien des jardins ouvriers et prétextent que leurs administrés, vivant toujours en plein air, ne portent aucun intérêt au sport et donc aux installations sportives. Le maire d'Arras dont les administrés sont particulièrement touchés par les problèmes de ravitaillement écrit :

La Municipalité se trouve obligée de vous soumettre, dans des conditions de hâte, qui ne dépendent pas d'elle, un programme de création d'aménagements de terrains de jeux et d'éducation physique à l'usage des enfants des écoles, qui peut entraîner une dépense considérable. Malgré l'objection des familles sinistrées et les besoins de terrain de culture, la Municipalité s'est efforcée de concilier tous ces besoins pour témoigner de sa bonne volonté et répondre à un appel qui lui est adressé

\footnotetext{
57 Gay Lescot 1991.
} 
évidemment dans le but de préparer, par la jeunesse, le relèvement rapide du pays ${ }^{58}$.

Les sinistrés et les élus ont donc des intérêts contradictoires. Jusqu'en 1950, les fortes réticences des villes, des communes et des sinistrés de la Zone Interdite retardent la mise en chantier des équipements et privent les agglomérations d'animations et d'activités ${ }^{59}$. En 1941, forte de 31500 habitants et de 6000 écoliers, la ville d'Arras subventionne, avec l'aide du CGEGS, sept terrains sportifs. Daniel Gilot, architecte de la ville, indique pourtant au maire que les matériaux laissés sur le chantier sont volés ou cassés, que certains poteaux en ciment armé posés pour établir les clôtures sont enlevés ou détériorés, et enfin que les scories disposées pour former le sol des pistes d'élan sont fouillées par des enfants et des habitants du quartier qui y cherchent le combustible ${ }^{60}$.

L'exploitation des infrastructures pose d'autres types de problèmes. En 1942, l'Abbé Cartel, directeur des œuvres d'Arras, demande au maire de la ville de bien vouloir réglementer l'usage des piscines afin de veiller particulièrement à la moralité et "d'éviter autant que faire se peut la possibilité de promiscuités fâcheuses $»^{61}$. Il souhaite qu'une période de temps soit réservée aux hommes et une autre aux femmes pour «qu'au moins certaines familles sachent qu'elles peuvent permettre la fréquentation de la piscine sans danger à leurs enfants, au moins à certains jours désignés ». La question de la mixité des lieux publics n'est pas nouvelle, surtout dans le domaine du sport où la différenciation des statuts masculin et féminin est, comme dans la société civile, très prégnante ${ }^{62}$. Longtemps les débats entre partisans d'une homogénéisation des pratiques et ceux d'une stricte séparation se sont confondus avec l'ensemble des conflits opposant les « laïcs » et les «confessionnels». L'affaiblissement dans

58 Maire d'Arras. Projet d'équipement sportif de la ville d'Arras, février 1941. AM d'Arras, 3R1.

59 Voldman 1997.

60 Daniel Gilot, architecte de la ville d'Arras agréé aux bâtiments publics et des communes au maire, le 28 juillet 1942. AM d'Arras, 3R4.

61 Diocèse d'Arras. Direction des œuvres. Abbé François Cartel : lettres à l'adjoint au maire d'Arras, 1 ${ }^{\text {er }}$ avril, 11 et 20 mai 1942. AM d'Arras, 3R4.

62 Barraud 1996. 
l'entre-deux-guerres et après la Seconde Guerre mondiale de ce type d'affrontement idéologique au profit du processus de «rationalisation» du sport et de l'éducation physique ne va pas résoudre le problème de la mixitée ${ }^{3}$. Au-delà des positions officiellement déclarées par les autorités religieuses, pour de nombreux groupes et pour le gouvernement de Vichy, la séparation des garçons et des filles reste la norme. Si la question de l'attribution d'horaires spécifiques aux femmes par des piscines municipales est toujours d'actualité, la demande de séparation entre les sexes ne repose pas exclusivement sur des bases religieuses. Sous Vichy, la pression exercée par les autorités allemandes provoque l'indignation du maire d'Arras qui regrette que la Kreiskommandantur refuse, pour pouvoir s'entraîner, de libérer le stade Degouve alors que celui-ci, doté d'une clôture, "assure la discrétion convenable pour les séances d'éducation physique des jeunes filles les après-midi $»^{64}$. Outre déplorer le mauvais état dans lequel les troupes d'occupation ont laissé la salle, le président du cercle de gymnastique et d'armes d'Arras indique que le local qui a été conçu «à une époque où notre sport était presque exclusivement masculin ne comporte qu'un vestiaire $»^{65}$, alors que le cercle compte désormais une section féminine. Par ailleurs, le seul terrain existant dans la ville de Douai (stade Demeny) est surexploité par deux sociétés sportives, par les établissements scolaires et par l'armée d'occupation. Sur incitation de l'inspecteur de l'éducation générale et sportive, le maire de Douai aménage cinq terrains scolaires provisoires d'éducation physique ${ }^{66}$. Cependant, en 1943, le sous-préfet constate que les terrains de la tour des Dames et

63 Clément 1996.

64 Kreiskommandantur Arras à Bayard, capitaine de la mairie d'Arras. Terrain du stade Degouve et de la salle de gymnastique municipale, le 15 avril 1943. AM d'Arras, 3R2. Pour la Kreiskommandantur, la répartition du service militaire ne permet pas de reporter le sport aux heures matinales.

65 Président du cercle de gymnastique et d'armes d'Arras au maire d'Arras, le 11 octobre 1941. AM d'Arras, 3R1.

66 Le sous-préfet au maire de Douai. Situation en matière d'équipement de sports de la ville de Douai, le 25 octobre 1941. AM de Douai, 3R20.

Académie de Lille, EGS. Direction départementale du Nord. L'inspecteur adjoint au maire de Douai, le 28 janvier 1942. AM de Douai, 3R20. 
du jardin des plantes sont laissés à l'abandon (feuilles mortes, portes ouvertes, terrain livré au public) ${ }^{67}$ et que les élèves de l'Institution Saint-Jean, sortent par-dessus les grillages et se suspendent aux branches d'arbres. Or, l'entretien des parcs est à la charge des municipalités ${ }^{68}$. En 1944, ce sont les difficultés de communications et les bombardements qui réduisent les réalisations, notamment à Lille ${ }^{69}$. Finalement, les obstacles (lenteurs administratives, manque de matériaux et de main d'œuvre, problème de communication et bombardements), les aléas (administrés qui refusent la suppression de cultures maraîchères, cohabitation entre les populations locales et les autorités allemandes) et les réticences, notamment des autorités religieuses locales et des autorités d'occupation allemandes qui nient toute requête même d'ordre moral, montrent la difficile mise en application de la doctrine sportive féminine. Les obstacles concernant les cadres de Vichy sont également pluriels.

Madame Champetier de Ribes, conseillère technique, déplore le contenu de formation (conférences et démonstration de gymnastique rythmique d'Irène Popart) dispensé au sein de l'Institut national d'éducation générale et sportive :

Nous ne sommes pas prêts encore à appliquer un programme d'une valeur idéale de gymnastique corrective, ni même éducative. Des erreurs sont commises et la propagande est assez mauvaise. Des praticiens dangereux pour une éducation physique aussi bien que pour une éducation tout court ont été attachés au Commissariat, ou protégés plus ou moins par lui (je pense à certaines méthodes rythmiques!) ${ }^{70}$.

67 Académie de Lille, EGS. Le directeur départemental de l'EGS du Nord au maire de Douai, le 17 novembre 1943. AM de Douai, 3R20.

68 Voldman $1997: 269-281$.

69 Secrétariat d'État à l'Éducation nationale. CGEGS. Direction de l'équipement sportif. Paris, le 15 janvier 1944. Les bombardements n'ont pas permis à la municipalité de Lille de bénéficier de la subvention du directeur de l'équipement sportif qui devait faciliter l'acquisition des terrains Faidherbe, Chevalier Français, Château Lemoine et de l'INEA. AM de Lille, 3R2/72.

70 Madame Champetier de Ribes, conseillère technique. Correspondances avec Monsieur le Colonel Bernard S/dir d'EGS. Vichy, le 27 janvier [1943?]. AN, CARAN, F44/42. 
Selon la conseillère, la méthode nationale présentée à la presse serait agrémentée de photos « désastreuses » qui nuisent à la renommée du CGEGS auprès des médecins et des mères de famille. Aussi, elle émet des réserves sur l'attitude des élèves :

J'ai été choquée de voir l'attitude "en liberté » des élèves, hommes et femmes, de l'école nationale de Paris. Je frémis quand je pense que ce sont là les futurs cadres pour l'Éducation de nos enfants! Je connais autour de moi de nombreuses familles de France qui, il y a peu d'années n'auraient pas cherché un «métier» pour leur fille, mais actuellement se trouvent justement dans une situation matérielle et dans un état d'esprit à le faire ${ }^{71}$.

Pour améliorer les conditions de recrutement des cadres, elle propose que l'école des hommes soit séparée de celle des femmes. Enfin, elle souligne que les éducatrices sont à l'origine de la véritable rénovation et que par conséquent une importance particulière doit leur être accordée :

C'est la femme comme mère qui guide la première partie de la vie de l'homme. Et c'est la femme comme femme qui souvent est la véritable responsable de la vie des hommes ! $!^{72}$.

Alors qu’à cette époque, Marie-Thérèse Eyquem souhaite que les personnels féminins remplacent progressivement les personnels masculins, la restitution d'extraits d'entretiens menés dans le cadre de notre recherche montre que, dans les centres régionaux d'éducation générale et sportive, les séances d'éducation physique sont majoritairement dispensées par des hommes :

Pendant la guerre c'était les bombardements. Tout était détruit. Je ne sais pas qui ils auraient pu envoyer dans les centres de formation, surtout chez les femmes. Les monitrices ont été formées beaucoup plus tard. C'était les grands gymnastes qui prenaient les enfants et ils progressaient comme ça quoi ${ }^{73}$.

71 Ibid.

72 Ibid.

73 Entretien avec Madame Dubois, 28 janvier 2004 à son domicile. Agée de 10 ans sous Vichy, elle est scolarisée à l'école municipale de Sallaumines. Sa mère l'inscrit au patronage où elle pratique la danse. Après la guerre, elle devient monitrice d'éducation physique dans les écoles puis développe la gymnastique volontaire dans sa ville natale. 
Parmi les personnes interrogées, il est courant de penser que la formation des cadres féminins sous Vichy a été particulièrement délicate voire inopérante. C'est ce qu'affirme Madame Guillemant, institutrice de Bully-les-Mines sous Vichy :

Je me souviens qu'en 1944, j'ai fait un stage de 6 semaines de gymnastique à Arras. C'était obligatoire pour notre formation. Notre directrice tenait beaucoup à ce que l'on réussisse mais moi je n'écoutais pas ce que disait le maréchal ${ }^{74}$.

Par ailleurs, le Commissariat général, qui veut éviter les cours mixtes, annule en novembre 1942 l'organisation des cours de formation des cadres féminins des associations sportives (monitrices à l'échelle cantonale) du CNMMA. En 1943, des cours régionaux d'aidesmonitrices d'associations sportives et des stages de monitrices de gymnastique harmonique et rythmique sont ouverts mais le besoin de moniteurs et de monitrices est toujours aussi pressant ${ }^{75}$. À l'École nationale d'éducation physique et sportive le commissaire général ajourne les épreuves orales et pratiques des examens et concours du professorat et moniteurs d'éducation physique et sportive de 1944 en raison des difficultés et des restrictions de toutes sortes causées par les bombardements anglo-américains ${ }^{76}$. Les instituts régionaux d'éducation physique, surtout ceux de Rennes, Lille et Besançon sont frappés par les bombardements et ne disposent plus de matériel. De plus, les troupes allemandes occupent leurs locaux. Certains instituts rencontrent des difficultés pour établir leurs emplois du temps et exploiter les infrastructures compte tenu du mauvais état des terrains (les Allemands gardent leurs bottes pendant les exercices). De plus, la suppression des trains et des autocars engendre des communications longues, coûteuses pour les élèves. La ligne de démarcation entre la

74 Entretien avec Madame Guillemant, 11 avril 2007 à son domicile. Diplômée de l'École Normale, elle est, sous Vichy, une jeune institutrice à Bully-les-Mines.

75 Lettre du directeur régional de l'éducation générale et des sports de l'académie de Lille du 9 janvier 1942. ADN, 1W770. Le directeur écrit: «Le besoin de moniteurs est toujours aussi pressant. Une lettre recommandée du 9 janvier vous signale à nouveau les besoins les plus impérieux ».

76 Ministère de l'Éducation nationale, CGEGS. Le Commissaire général aux Sports au Directeur du centre national d'éducation générale. Paris, le 19 juin 1944. 
Zone Interdite et la Zone Occupée limite les déplacements. Vivant dans la crainte des bombardements, les étudiants, sous alimentés, sont inaptes à l'effort physique. Enfin, la fermeture des piscines, le manqué de vêtements et l'insuffisance des crédits affectés, comme à Lille, contrarient le fonctionnement et la fréquentation des instituts qui redoutent la concurrence des centres régionaux ${ }^{77}$.

Finalement, les obstacles conjoncturels et structurels (restrictions liées aux bombardements, manque de matériel, difficulté de planification des enseignements dans les centres et instituts, occupation et dégradation des locaux par les troupes d'occupation, moyens de communications limités) rendent le bilan du Commissariat général discutable. Par ailleurs, les réserves émises sur le contenu de formation, la propagande et l'attitude des cadres féminins, le recrutement limité des candidates, le problème que pose la mixité et les réserves des parents ${ }^{78}$, des médecins et des éducatrices ${ }^{79}$, peu convaincus de l'intérêt du sport et de l'éducation physique pour les femmes et les jeunes filles, le discréditent davantage.

\section{La sportive française sous Vichy : entre le dire et le faire}

Pour les féministes, le sport est un lieu d'émancipation, de libération et de conquête égalitaire des femmes; mais les médecins ne l'acceptent que pour des raisons natalistes, et recommandent une pratique modérée pour les mêmes raisons. Au masculin, l'activité physique semble favoriser l'homophobie et des modèles de virilité très stéréotypés et très traditionnels ${ }^{80}$. Ainsi, le sport "demeure idéologiquement organisé selon des critères explicites ou diffus d'appartenance sexuelle où chacun et chacune est jugé et classé selon

77 Éducation nationale, CGEGS. Monsieur J.J. Chevallier, directeur de l'EGS. Paris le 2 mars 1942. AN, CARAN, F44/40.

78 Le comité de l'Union douaisienne porte à la connaissance des familles que des séances d'éducation physique pour filles ont lieu tous les jeudis. Les séances sont particulières aux filles et les parents peuvent être assurés qu'une bonne tenue et une parfaite discipline seront maintenues parmi les élèves.

79 Procès verbal de l'USSU, le 19 juin 1944 au CGEGS. Rapport moral 1943-1944, p. 13-14. AN, CARAN 69AJ4.

$80 \quad$ Salle 2006. 
ses goûts, ses pratiques, ses comportements ou ses formes corporelles $»^{81}$. Sous Vichy, au-delà des rôles que le commissariat et Marie-Thérèse Eyquem voulaient assigner aux femmes et au sport en particulier, la pratique féminine a obtenu une certaine reconnaissance, qui n'avait pas été accordée jusque-là "malgré les prouesses et combats de certaines $»^{82}$. Sans doute, les contradictions internes, certains événements, les rigueurs de l'Occupation et des occupants ont contrarié l'expression de cette forme de féminité. Mais cette féminité qui a pu se manifester dans la Zone Interdite où la main allemande contrôle l'espace social, atteste à la fois d'une subordination et d'une «culture d'évasion » ${ }^{83}$ par rapport aux mots d'ordre du régime. On mesure ici toute l'ambivalence du sport, pouvant servir à la fois de vecteur idéologique, de «refuge » mais aussi d'espace de résistance pour les individus. Après la guerre, certains dirigeants sportifs ne seront pas inquiétés. Marie-Thérèse Eyquem, devenue inspectrice à la Libération, progressa dans la hiérarchie jusqu'à devenir inspectrice principale de la Jeunesse et des Sports en 1961.

\section{Bibliographie}

ARNAUD Pierre \& Thierry TeRret (dir.), 1996, Histoire du sport féminin, tome 1, Histoire et Identité, tome 2, Sport masculin - sport féminin: éducation et société, Paris, L'Harmattan.

ARNAUD Pierre \& James Riodan, 1998, Sport et relations internationales (1900-1941). Les démocraties face au fascisme et au nazisme, Paris, L'Harmattan.

Arnaud Pierre, Terret Thierry, Gros Pierre \& Jean-Philippe Saint-Martin (dir.), 2002, Le sport et les Français pendant l'occupation, 1940-1944, Paris, L'Harmattan.

AzÉMA Jean-Pierre, 1979, De Munich à la Libération, 1938-1944, Paris, Points Seuil.

AZÉMA Jean-Pierre \& François BÉDARIDA, 1992, Vichy et les Français, Paris, Fayard.

—, 1993, La France des années noires, Paris, Seuil, 2 volumes.

81 Roger \& Terret $2005: 9$.

82 Zancarini-Fournel $2005: 130$.

83 Rousso 1990, 1992, 1994. 
BARRAUD Nicole, 1996, «Histoire de la natation synchronisée. La conquête de l'eau par les femmes ", in Pierre ARnAUd \& Thierry Terret (dir.), Histoire du sport féminin. Histoire et identité, Tome 1, Paris, L'Harmattan, p. 25-37.

CAILlois Roger, 1967, Encyclopédie de la Pléiade, Jeux et Sports, Paris, Édition Gallimard.

CALLÈDE Jean-Paul, 1998, « La France sportive de Vichy (1940-1944). L’ordre contre la démocratie ", in Sport et démocratie, Paris, Assemblée nationale, p. 67-70.

CASTAn Vicente Florys, 2009, «Marie-Thérèse Eyquem. Du sport à la politique, parcours d'une féministe », L'Ours, p. 54.

ClÉMENT Jean-Paul, 1996, «Pratiques corporelles féminines, différenciation sexuelle et gestion de la mixité en EPS (1945-1980)", in Pierre ARNAud \& Thierry TERRET (dir.), Histoire du sport féminin. Sport masculin - sport féminin : éducation et societé, tome 2, Paris, L'Harmattan, p. 213-226.

Dejonghe Étienne \& Yves Le Maner, 1998, Le Nord Pas-de-Calais dans la main allemande (1940-1944), Lille, La Voix du Nord Éditions.

Delaplace Jean-Michel, Treutlein Giselher \& Gerhard SpiTZER, 1994, Le sport et l'education physique en France et en Allemagne : contribution à une approche sociobistorique des relations entre les deux pays, Édition AFRAPS.

EYQUEM Marie-Thérèse, 1944, La femme et le sport, J. Susse, coll. « Tous les Sports ».

—, 1967, «La gymnastique et le sport féminin », in Roger CailLois, Jeux et Sports, Paris, Gallimard, coll. « Pléiade Encyclopédie » n²3, p. 1826-1838.

FAURE Christian, 1989, Le projet culturel de Vichy. Folklore et révolution nationale, 19401944, Lyon, Centre régional de publication de Lyon, Paris, Presses du CNRS.

FOnTAINE Thomas, 2009, Chronologie : répression et persécution en France occupée 19401944, Encyclopédie en ligne des violences de masse.

Gay LesCOT Jean-Louis, 1991, Sport et éducation physique sous Vichy, Paris, Perrin.

LABORIE Pierre, 1990, L'opinion française sous Vichy, Paris, Éditions du Seuil.

LEBRUN Bernard \& Yvon LEZIART, 2005, «La formation des professeures d'éducation physique à l'ENEPS, 1943-1950 : analyses à partir de la revue Les notes techniques de l'ENSEP », in Jean-Philippe SAINT-MARTIN \& Thierry TERrET, Sport et genre, Apprentissage du genre et institutions éducatives, Volume 3, Paris, L'Harmattan, p. 263-282.

LIOTARD Pierre \& Thierry TERRET, 2005, Sport et genre. Excellence féminine et masculinité bégémonique, Paris, L'Harmattan.

Louveau Catherine, 2006, "Inégalité sur la ligne de départ: femmes, origines sociales et conquête du sport », Clio. Histoire, femmes et sociétés, 23, p. 119-143.

MuEL-Dreyfus Francine, 1996, Vichy et l'éternel féminin, Paris, Éditions du Seuil. 
MunOZ Laurence, 2002, «Marie-Thérèse Eyquem (1913-1978) au moment de l'Occupation ", in Pierre ARnAud, Thierry Terret, Pierre Gros \& Jean-Philippe SAINT-MARTIN (dir.), Le sport et les Français pendant l'Occupation (1940/1944), tome 2, Paris, L'Harmattan, p. 65-72.

Paxton Robert, 1973, La France de Vichy, 1940-1944, Paris, Seuil.

PesChAnSKI Denis, 1997, Vichy 1940-1944, contrôle et exclusion, Bruxelles, Complexe.

RAUCH Antoine, 1988, Vacances et pratiques corporelles. La naissance des morales du dépaysement, Paris, PUF.

Rioux Jean-Pierre \& Jean-François SIRINELLI, 1998, Histoire culturelle de la France. Les temps des masses, Paris, Le Seuil.

Roger Anne \& Thierry Terret (dir.), 2005, Sport et genre. Objets, arts et médias, Paris, L'Harmattan.

Rousso Henry, 1992, Les Années noires : vivre sous l'Occupation, Paris, Gallimard.

Rousso Henry \& Éric CONAN, 1990, Le syndrome de Vichy : de 1944 à nos jours, Paris, Le Seuil.

—, 1994, Vichy, un passé qui ne passe pas, Paris, Fayard.

Ruffin Raymond, 2004, Violette Morris : la byène de la Gestapo, Paris, Le Cherche Midi.

SAINT-MArTin Jean-Philippe \& Thierry Terret (dir.), 2005, Sport et genre. Apprentissage du genre et institutions éducatives, Volume 3, Paris, l'Harmattan.

Salle Muriel, 2006, compte rendu de Thierry Terret et al. (dir.), Sport et genre, Clio Histoire, femmes et sociétés, 23.

TERRET Thierry, 2005, Sport et genre. La conquête d'une citadelle masculine, Paris, L'Harmattan.

THIERY Laurent, 2007, «Les spécificités de la répression dans le Nord Pas-de-Calais, "zone rattachée au commandement militaire allemand de Bruxelles" ", in La répression en France 1940-1945, Actes du colloque international de Caen, 8-10 décembre 2005, Caen, Éditions du CRHQ.

VOLDMAN Danièle, 1997, La reconstruction des villes françaises de 1940 à 1954, histoire d'une politique, Paris, L'Harmattan.

ZanCARINI-Fournel Michelle, 2005, Histoire des femmes en France XIXe-XXe siècle, Rennes, Presses Universitaires de Rennes, coll. « Didact. Histoire ». 